Instituto Internacional de Investigación y Desarrollo Tecnológico Educativo INDTEC, C.A.

DOI: https://doi.org/10.29394/Scientific.issn.2542-2987.2021.6.20.17.311-326

OAI-PMH: http://www.indteca.com/ojs/index.php/Revista Scientific/oai

Ensayo Original / Original Essay

\title{
Beneficios del trabajo en equipo durante la formación académica entre áreas de la salud
}

\author{
Autor: Diego Alvarado Melitón \\ Hospital Psiquiátrico Fray Bernardino Álvarez, HPFBA \\ ocupacional fray@outlook.com \\ Ciudad de México, México \\ https://orcid.org/0000-0002-4116-885X
}

\section{Resumen}

El trabajo en equipo facilita la interacción entre compañeros para la toma de decisiones y resolución de problemas o tareas que causan cierta dificultad, cuya teoría es basada de acuerdo con Ayoví-Caicedo (2019), por otro lado se hace mención sobre el liderazgo educativo siendo de suma importancia según lo menciona Sierra (2016), ya que es la representación institucional de cada alumno y expresada en calidad educativa y humanismo, en este sentido Rando (2016), nos hace énfasis en el trabajo en equipo multidisciplinario para tener una buena comunicación entre profesionales, se hace referencia acerca del liderazgo conciliador destacando a Goleman (2013), en cuanto a relaciones interpersonales, son fundamentales conforme a Hernández e Hinojos (2019), finalmente, teniendo como objetivo principal lograr la enseñanza del trabajo en equipo con las diferentes disciplinas del área de la salud durante la formación académica en las instituciones de educación superior y promoviendo una transversalidad en el proceso de enseñanza aprendizaje, mediante la vinculación entre ámbito académico e institución de salud pública, sin embargo al estimular las interacciones sociales con la implementación de estrategias didácticas basadas en el trabajo de equipo en fases tempranas de la formación académica puede beneficiar a los estudiantes en su ámbito laboral.

Palabras clave: interacción social; salud pública; estrategias educativas; humanismo; liderazgo.

Código de clasificación internacional: 3204.03 - Salud profesional.

Cómo citar este ensayo:

Alvarado, D. (2021). Beneficios del trabajo en equipo durante la formación académica entre áreas de la salud. Revista Scientific, 6(20), 311-326, e-ISSN: 2542-2987. Recuperado de: https://doi.org/10.29394/Scientific.issn.2542-2987.2021.6.20.17.311-326

Fecha de Recepción: 13-01-2021
Fecha de Aceptación: 30-03-2021
Fecha de Publicación: 05-05-2021 


\title{
Benefits of teamwork during academic training between health areas
}

\begin{abstract}
Teamwork facilitates interaction between colleagues for decisionmaking and resolution of problems or tasks that cause certain difficulty, whose theory is based according to Ayoví-Caicedo (2019), on the other hand, mention is made of educational leadership being of utmost importance as mentioned by Sierra (2016), since it is the institutional representation of each student and expressed in educational quality and humanism, in this sense Rando (2016), emphasizes multidisciplinary teamwork to have good communication Among professionals, reference is made about conciliatory leadership, highlighting Goleman (2013), in terms of interpersonal relationships, they are fundamental according to Hernández and Hinojos (2019), finally, having as main objective to achieve the teaching of teamwork with the different disciplines of the health area during academic training in higher education institutions and promoting a transversal identity in the teaching-learning process, through the link between the academic environment and the public health institution, however, by stimulating social interactions with the implementation of teaching strategies based on teamwork in the early phases of academic training, it can benefit students. students in their work environment.
\end{abstract}

Keywords: social interaction; public health; educational strategies; humanism; leadership.

International classification code: 3204.03 - Occupational health.

\section{How to cite this essay:}

Alvarado, D. (2021). Benefits of teamwork during academic training between health areas. Revista Scientific, 6(20), 311-326, e-ISSN: 2542-2987. Recovered from: https://doi.org/10.29394/Scientific.issn.2542-2987.2021.6.20.17.311-326

Date Received: 13-01-2021
Date Acceptance: 30-03-2021
Date Publication: 05-05-2021 


\section{Introducción}

El presente ensayo tiene como finalidad dar a conocer los beneficios de la enseñanza del trabajo en equipo en las licenciaturas del área de la salud durante la formación académica, ya que es fundamental para el desarrollo de habilidades sociales, profesionales y personales, tomando en cuenta que los alumnos universitarios que están en el proceso formativo deben mantener relaciones sociales de las que van a depender para una atención integral y de calidad a los usuarios que hacen uso de los servicios de salud pública, formando equipos de trabajo interdisciplinario, multidisciplinario y transdisciplinario.

En el área de la salud es de gran importancia mantener una relación de trabajo en equipo en los niveles de atención primaria, secundaria y terciaria, motivo por el cual al saber trabajar con varios integrantes de diferentes áreas profesionales y mantener buenas relaciones sociales entre compañeros por lo tanto se ofrece una mayor calidad de atención hacia los usuarios, es por eso que cuando los estudiantes finalicen sus estudios profesionales y lleguen a desempeñarse en el ámbito laboral es más fácil crear relaciones interpersonales y multidisciplinares entre profesionales.

Sin embargo, en la actualidad es evidente una mala relación entre compañeros de trabajo de las áreas de salud, esto provoca malas praxis en los servicios de atención sociosanitaria, entre los principales factores se encuentran las interferencias emocionales y sociales que se ven reflejadas en los procedimientos rutinarios ante los usuarios que acuden para una atención oportuna, sin embargo, estos procesos son muy lentos y con dificultades a su abordaje, por lo que es necesario hacer conciencia hacia la institución educativa la cual debe tener en claro que se están formando estudiantes con ética, moral y humanismo.

Finalmente, el objetivo es lograr una enseñanza de trabajo en equipo con las diferentes disciplinas del área de la salud durante la formación 
académica en las instituciones de educación superior y proporcionar elementos que promuevan una transversalidad en el proceso de enseñanza aprendizaje mediante una vinculación entre el ámbito académico y de instituciones de salud pública para fortalecer relaciones sociales entre dichas áreas de la salud, destacar los beneficios que se obtienen al trabajar en equipo, además de favorecer el liderazgo, empatía, capacidad de análisis, liderazgo, compañerismo, comunicación y respeto, entre otros beneficios adicionales mediante el uso de estrategias didácticas que sean impartidas por docentes expertos en el manejo de grupos colectivos y por consecuente garantizar un servicio de calidad y humanismo a los servicios de atención sanitaria.

\section{Desarrollo}

El trabajo en equipo es fundamental en ciertas áreas laborales debido a que permite una mayor solución de problemas teniendo en cuenta que los individuos al desempeñar un puesto laboral, logran cumplir con una tarea satisfactoriamente en tiempo y forma correcta, la cooperación de más compañeros de trabajo facilita tareas que tienen una mayor complejidad para llevar a cabo, siendo notorio en los ámbitos hospitalarios, las funciones a desempeñar de cada profesional de la salud tienen cierto grado de complejidad.

Durante la formación académica de los estudiantes en las áreas de salud, es un factor determinante enseñar y dominar el trabajo en equipo, debido a que no solo es llevar a cabo un proceso de enseñanza-aprendizaje, también es la de proporcionar herramientas que permitan desarrollar ciertas habilidades personales, sociales y profesionales entre otros compañeros con áreas a fines, cuya finalidad es la de hacer más eficientes a la hora de poner en práctica los procesos de atención al usuario que requieren en instituciones de salud. 
Por lo tanto, en las instituciones de educación superior deberían implementar materias que compartan la transversalidad del conocimiento enfocado a escenarios de simulación real para el ámbito de salud, el cual facilitaría un mayor desempeño a los universitarios, debido a que crearían una conciencia de la importancia del trabajo en equipo, así como los procesos requeridos en un ambiente real de trabajo, por lo tanto, en palabras de AyovíCaicedo (2019):

[...] El Trabajo en Equipo es un concepto que encierra en sí mismo la cohesión, la unión y la transformación de una organización, es sinónimo de productividad, competitividad y logro de objetivos, basándose fundamentalmente en la necesidad de mejoramiento continuo y cambios que se dan dentro de las estructuras organizacionales para dar cumplimiento a la misión y visión establecida y que permita orientar el comportamiento de los individuos con el fin de alcanzar la máxima calidad y productividad (pág. 60).

Igualmente, sería recomendable que se integraran planes de trabajo en las instituciones de educación superior cuya finalidad es la de poner en práctica la toma de decisiones, solución de problemas, trabajo colaborativo, liderazgo en los estudiantes que inician en el ámbito de la salud además de ejemplificar el cumplimiento de la misión y visión de su profesión, por lo tanto, la ideología de compañerismo se puede ir mejorando y dejar de lado los egos profesionales a futuro desde etapas tempranas permitiendo la inclusión del apoyo colectivo.

En cuanto a la institución educativa a nivel superior es necesario que reconozca que tipo de liderazgo ejerce a través de la formación académica de sus estudiantes y de que manera representa el proceso de enseñanzaaprendizaje, además de tomar en cuenta la misión y visión de la institución, esto se realiza por medio de quien dirige y su intervención en la misma, además de ser modelo ejemplar para los que imparten clases en sus aulas y que favorecen las funciones sociales que se trasmiten a sus alumnos 
notándose en sus egresados mediante la calidad educativa y humanismo que permite una identidad institucional a cada alumno en formación como lo menciona Sierra (2016):

El Liderazgo educativo se establece con la dinámica de la acción formativa de las personas fundamentada en ideales filosóficos, y, por consiguiente, en la formación integral. En cuanto a lo estratégico y técnico con esencia sostenible frente a la comprensión, intervención y trasformación de la cultura, lo ambiental y lo social. Así, se permite la proyección del ser, hacia el sentido de la educación humana, la cual se refleja en todas sus dimensiones y escenarios los cuales garantizan mejorar la calidad de vida, en lo profesional y personal, sustentado sobre la ética y en la valoración de los recursos de las generaciones futuras (pág. 114).

Por lo que refiere al trabajo de equipo en las instituciones de salud se torna una situación laboriosa y con una sobrecarga laboral que presenta el personal, evidenciados por medio de fatiga, estrés, mala toma de decisiones, diagnósticos equivocados, errores de medicación, conflictos entre compañeros e incluso a tal grado de llegar a eventos adversos, errores médicos, etc., teniendo esto en evidencia, se hace énfasis en que al intentar enfrentar el problema de raíz se puede beneficiar a las relaciones sociales en una institución de salud por medio del fomento para trabajar en equipo desde las instituciones formadoras de educación superior.

Así pues, para Rando (2016): "el trabajo en equipo multidisciplinario aumenta la seguridad de los pacientes, minimiza los errores médicos, mejora la satisfacción y el rendimiento del personal de salud [...]" (pág. 59). Por lo tanto, es necesario tener una buena comunicación entre los profesionales de la salud de las diferentes áreas, además de ser la base esencial de todo proceso que se requiera en la atención a los usuarios de servicios de salud, entre las características más relevantes que brinda un trabajo en equipo multidisciplinario son, organización, satisfacción, innovación y la calidad de 
atención que se brinda hacia los usuarios.

Igualmente, en el trabajo de equipo interdisciplinario conformado por profesionales específicos de salud, se debe llevar un proceso acorde a las necesidades de los usuarios y una vez terminado su objetivo específico, se deberá delegar las funciones específicas a otro profesional de la salud, dando a conocer los esfuerzos realizados por sus compañeros ante los usuarios, pero en ciertas ocasiones los profesionales de la salud no dan este reconocimiento debido a la poca empatía que hay entre ellos y remarcando que en dichos procesos se hace una acción aislada que complica el trabajo en equipo.

Parte fundamental del equipo interdisciplinario es la de permitir la participación del equipo de profesionales para llegar a un fin común que permita el beneficio al usuario pero teniendo en cuenta que todo depende del funcionamiento y la participación de los integrantes del equipo, cabe mencionar que lo más importante es la responsabilidad con la cual deben de actuar en dichos procesos y el intercambio fluido de opiniones en una forma que permita la construcción de intervenciones adecuadas y el logro de los objetivos planteados de las integrantes del equipo.

En consecuencia el trabajo en equipo transdisciplinario es el punto más difícil de vencer al trabajar en equipo porque ahora los aspectos que más resaltan son un liderazgo, comunicación, autoconocimiento y empatía dejando de lado los celos profesionales y delirios de omnisapiencia, entre otros y permiten el desempeño de un liderazgo adecuado que fomente la comunicación entre todos los profesionales de la salud logrando habilidades sociales, profesionales y personales, además de cumplir con éxito el objetivo general en los usuarios que acuden a las instituciones de salud.

Con respecto a la enseñanza del trabajo de equipo en las instituciones de educación superior es necesario que el docente asuma el rol de líder ya que el será quien asuma el buen funcionamiento del grupo y logre un aprendizaje digno y significativo en cada integrante de tal manera que todo 


\section{Ensayo Original / Original Essay}

conocimiento teórico y práctico pueda ser empleado con una planificación adecuada, un liderazgo optimo y una intervención que promueva objetivos alcanzables donde todo el equipo se motive con una facilidad intrínseca y se vea reflejado en los participantes activos.

Se sabe que hay diferentes tipos de liderazgo, entre los que destacan el autoritario, conciliador, democrático, ejemplarizante, coach y coercitivos, siendo el ideal para el área de la salud el liderazgo conciliador de acuerdo con Goleman (2013): "el líder conciliador busca que los trabajadores estén contentos y exista armonía entre ellos. Su forma de gestionar consiste en crear fuertes vínculos emocionales y luego recoger los frutos de ese planteamiento, en concreto una enorme lealtad" (pág. 171). Por lo tanto, este liderazgo además de generar armonía también brinda la opción de incrementar la moral de las personas, algo muy útil para los estudiantes en formación académica. En efecto, las áreas de la salud son involucradas en la parte moral, ética y humanista desde la formación académica, para Hardy-Pérez, Rovelo-Lima (2015):

La moral se refiere a las creencias y prácticas de primer orden que implican un conjunto de juicios establecidos que originan y siguen los individuos, las familias o sociedades para orientar sus acciones y comportamiento en la vida diaria. Estos códigos morales pueden servir de referencia a instituciones, grupos y sociedades enteras, impregnando procesos esenciales de socialización y de pautas de actuación (pág. 80).

Mientras tanto, la ética es una reflexión de la moral y que conlleva a diferenciar entre lo bueno y lo malo, de manera que se garantizar la práctica de valores en los estudiantes del área de la salud o en su caso implementar nuevas estrategias para complementar la formación en dichos valores que sean parte del perfil de egreso de todo universitario, en conformidad con Izaguirre (2019):

Comprender la importancia de la formación ética de los 
estudiantes de medicina, obliga analizar la realidad actual de la educación médica para determinar qué criterios éticos y morales se le debe inculcar a los estudiantes durante su formación para que sean excelentes profesionales con responsabilidad ética y jurídica (pág. 508).

De ahí que los estudiantes desde su primer abordaje y contacto con los usuarios, deben comprender cuáles serán sus derechos y obligaciones donde se pone en juego los valores y también a los demás profesionales de la salud en el momento de brindar un servicio con calidad y humanismo, por ejemplo, tenemos la revisión cotidiana de signos vitales y medidas somatométricas por parte del personal de enfermería, valoración y tratamiento de una enfermedad realizada por personal médico y de aquí parte su envió hacia las diferentes áreas de rehabilitación, psicología y nutrición, si el usuario lo requiere.

En cuanto a la enseñanza de las relaciones sociales es fundamental favorecer la estimulación de relaciones interpersonales en las aulas mediante la intervención por estrategias que deberá llevar a cabo el docente y tener en cuenta el liderazgo, empatía, y la regulación de emociones para evitar interferencias sociales a futuro, si bien algo que es muy notorio en diferentes áreas de la salud es la mala relación que existen entre compañeros cuando aún están en plena formación académica, si bien un ejemplo claro a continuación que señalan las autoras Hernández e Hinojos (2019):

Construir relaciones interpersonales entre enfermeros es un desafío grande, ya enfermería es un contexto de alta complejidad, en el que lamentablemente muchas veces se presentan intereses individualistas, alta competitividad y desconfianza entre compañeros, siendo entonces una sociedad muy exigente; sin embargo, es algo de vital importancia, pues lo anterior llevaría a una disminución del interés colectivo y social, pudiendo terminar en grupos de profesionales de enfermería llenos de egoísmos y miedo al prójimo (pág. 43).

Sin embargo, enfermería no es la única área que presenta dichos 
intereses en sus estudiantes, este problema es en general para las demás profesiones, ya que como es bien sabido, cada institución educativa ofrece competencias educativas las cuales son necesarias a nivel superior, pero en realidad no se llevan a cabo estos conocimientos en su práctica diaria, o simplemente se le da más importancia al dominio del conocimiento teórico y se deja de lado las habilidades y destrezas que puedan favorecer las acciones sociales en conjunto, por eso es importante saber cómo es el plan de trabajo y quienes son los docentes que facilitan estas herramientas a los futuros profesionales.

Conviene resaltar que la idea principal para las instituciones de educación superior es la de generar e intercambiar un conocimiento que ya está presente pero no se le da la importancia necesaria por parte del área de salud ni autoridades a cargo de las mismas, dejando de lado la vinculación entre ambas instituciones y cuya aportación es la de modificar el plan de trabajo y hacer una transversalidad entre otras instituciones para promover líneas estratégicas de generar un conocimiento adicional y la de fomentar el trabajo en equipo a su vez permitir una mayor beneficencia en las relaciones con otros estudiantes del área de la salud.

En cuanto a las relaciones multidisciplinares es más complejo para nuestros estudiantes, ya que se debe contar con cierta experiencia para hacer frente a un problema con cierto grado de dificultad, en el cual la intervención por cada área de la salud y se demuestra, en el área de rehabilitación al proponer un plan de trabajo para el usuario en donde se pueda llevar a cabo una intervención y mantener una máxima comunicación con otros profesionales, por eso es esencial que desde la formación académica se proporciones las herramientas necesarias por parte del docente a cargo, el cual tiene que promover estrategias didácticas relacionadas al conjunto colectivo, tomando en cuenta la visión, organización, normas, roles, responsabilidades finalmente la evaluación del conocimiento adquirido. 
Por esto se deben buscar estrategias efectivas que permitan llevarse a cabo en las aulas y supervisadas por docentes que suelen trabajar en equipo, cabe mencionar que no cualquier docente pude llevar a cabo este tipo de estrategias grupales, se busca que el docente pueda desarrollar en los alumnos las competencias cognitivas, afectivas y sociales que demanda el trabajar en equipo, se hace la sugerencia del uso de las diferentes estrategias didácticas, como el taller reflexivo, aprendizaje basado en proyectos, estudio de casos, trabajo colaborativo, aprendizaje basado en problemas, aprendizaje basado en desafíos, practicas externas, debates, juego de roles, técnica del rompecabezas, modelo de Harvard.

Debido a la negatividad que se proyecta en diferentes ámbitos al trabajar en equipo desfavorece las relaciones sociales y denotando la falta de confianza y ausencia en la integración y aportación de ideas que puedan ser de utilidad, además de afrontar conflictos sumamente caóticos y la toma de decisiones inadecuadas, además de provocar roces emocionales con otros integrantes, compañeros, profesionales y usuarios, por consiguiente una falta de responsabilidad en las tareas que tenga que desarrollar y un poco atención a quien recurre a estos servicios que se brindan, a todo esto cabe mencionar que al ponerse en juego estos factores se desarrolla el pesimismo por parte de los integrantes del equipo.

Por el contrario, al trabajar en equipo, los estudiantes serán más conscientes de sus alcances y no cerrarse de manera individual, reconociendo los beneficios adicionales que se obtienen cuando se hacen en conjunto y asimilando la idea de lograr objetivos en conjunto y de manera favorecer las habilidades sociales, esenciales para relacionarse con otras personas y otros profesionales, respetando las decisiones y aportaciones dentro del conjunto colectivo, las relaciones profesionales, esenciales dentro de la formación del ámbito laboral y promoviendo experiencia para poner en práctica en otros contextos y las relaciones personales de cada estudiante que se adquiere, 
referente a la empatía, compañerismo, respeto y liderazgo.

Mientras tanto las instituciones de educación superior tengan la accesibilidad para notar las dificultades que se viven actualmente en los centros e instituciones que brindan atención de salud para trabajar en equipo y se mantenga una transversalidad en sus áreas de conocimiento dentro de las cuales, algunas materias brindan la posibilidad de hacer un conocimiento articulado; de la salud maternal, infantil, ambiental, mental, nutrición, prevención de accidentes, adicciones, violencia, primeros auxilios, actividad física, entre otras, siendo una oportunidad ideal para promover estilos de vida saludables en las instituciones y en los diferentes contextos socioeconómicos de la población.

Cabe mencionar que cada área de la salud cuenta con los conocimientos necesarios para hacer posible una intervención en conjunto y facilitar objetivos durante las practicas que se llevan a cabo es por eso la importancia del trabajo en equipo, ya que cada estudiante aporta nuevas ideas que son de gran utilidad hacia los demás integrantes haciendo que la perspectiva grupal mantenga el compromiso hacia los usuarios, donde a partir de necesidades exista la claridad de las funciones específicas de intervención por parte de los estudiantes y así poder asignar un líder que sea capaz de mantener y llevar a cabo un objetivo general que en este caso es el de brindar atención de calidad y con humanismo.

Es posible que al finalizar su formación académica los estudiantes tendrán una mayor empatía, una capacidad de análisis, liderazgo, compañerismo, comunicación y respeto, resolución de problemas, pero principalmente la sinergia que se pondrá en práctica con otros compañeros de trabajo, aunque si se emplearan estrategias didácticas que beneficien el trabajo colectivo y la aportación de planes educativos que promuevan la trasversalidad entre las diferentes instituciones educativas de educación superior en áreas de salud los resultados pueden ser positivos en nuestras 
nuevas generaciones en formación académica y promoviendo el humanismo.

\section{Conclusiones}

El trabajo en equipo es esencial en el ámbito laboral para cumplir exitosamente con tareas que implican una mayor dificultad por que facilitan la cooperación, toma de decisiones y la intervención entre profesionales, mas no así se limita para el ámbito hospitalario, es de gran utilidad su implementación en otros sectores debido a sus beneficios y los resultados que se obtienen al ponerse en práctica.

Para el ámbito del área de la salud es importante tener en cuenta que su enseñanza debe realizarse desde las instituciones de educación superior y no solo enfocarse en procesos de enseñanza-aprendizaje con mayor atención a contenidos conceptuales de lo contrario solo se fomenta a trabajar de forma aislada y que cause aversión a trabajar en colaboración con otros profesionales a futuro.

El liderazgo educativo es el reflejo de la formación académica que brinda la institución educativa hacia los estudiantes que durante la etapa formativa son empapados por la adquisición de valores, ética y el aprendizaje de las relaciones sociales que van de la mano con su perfil de egreso para desenvolverse en ámbitos laborales.

Las malas relaciones sociales y profesionales dentro y fuera de las instituciones tanto educativas como de salud, provocan sobrecarga en sus actividades arruinando la praxis profesional y provocando una serie de eventos adversos que tiene un desenlace nada agradable y cerrándose a un crecimiento personal, profesional y social.

El trabajo en equipo ya sea interdisciplinario, multidisciplinario y transversal permite desarrollar habilidades sociales, profesionales $y$ personales que favorecen a una mejor comunicación durante y después de la formación académica debido a que se brindan herramientas muy útiles que se 
pueden aplicar en conjunto con otros profesionales beneficiando una mejor atención con calidad, empática y con mayor humanismo.

La transversalidad entre las instituciones de educación superior y las instituciones de salud es fundamental para lograr un puente de conocimiento y necesidades para implementar nuevas competencias que demanda la actualidad y permitir su aplicación en dichos campos clínicos y hacer una simbiosis del conocimiento beneficiando a ambos sectores.

\section{Referencias}

Ayoví-Caicedo, J. (2019). Trabajo en equipo: clave del éxito de las organizaciones. Fipcaec, 4(19), 58-76, e-ISSN: 2588-090X. Recuperado de: https://doi.org/10.23857/fipcaec.v4i10.39

Goleman, D. (2013). Liderazgo: el poder de la inteligencia emocional. 1. ${ }^{\text {a }}$ edición, ISBN: 978-84-9019-432-4. Barcelona, España: Ediciones B, S.A.

Hardy-Pérez, A., Rovelo-Lima, J. (2015). Moral, ética y bioética. Un punto de vista práctico. Medicina e Investigación, 3(1). 79-84, e-ISSN: 22143106. Recuperado de: https://dx.doi.org/10.1016/j.mei.2015.02.007 Hernández, W., \& Hinojos, E. (2019). Relaciones interpersonales entre enfermeros para su bienestar. Revista Iberoamericana de Educación e Investigación en Enfermería, 9(4), 40-7, e-ISSN: 2174-6915. Madrid, España: Asociación Latinoamericana de Escuelas y Facultades de Enfermería (ALADEFE).

Izaguirre, M. (2019). Importancia de la formación ética de los estudiantes de medicina. Anales de la Facultad de Medicina, 80(4), 507-510, eISSN: 1609-9419. Recuperado de:

https://dx.doi.org/10.15381/anales.v80i4.16974

Rando, A. (2016). Trabajo en equipo: ¿Es posible formar equipos médicos expertos a partir de profesionales expertos?. Revista Médica del 
Instituto Internacional de Investigación y Desarrollo Tecnológico Educativo INDTEC, C.A.

DOI: https://doi.org/10.29394/Scientific.issn.2542-2987.2021.6.20.17.311-326

OAI-PMH: http://www.indteca.com/ojs/index.php/Revista Scientific/oai

Ensayo Original / Original Essay

Uruguay, 32(1), 59-67, e-ISSN: 1688-0390. Recuperado de:

http://www.scielo.edu.uy/scielo.php?script=sci arttext\&pid=S1688-

$\underline{03902016000100008}$

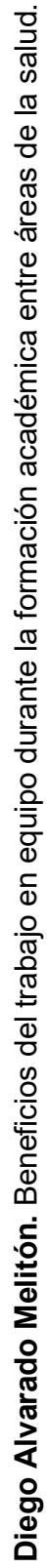

Sierra, G. (2016). Liderazgo educativo en el siglo XXI, desde la perspectiva del emprendimiento sostenible. Revista Escuela de Administración de Negocios, (81), 111-128, e-ISSN: 2590-521X. Recuperado de: https://doi.org/10.21158/01208160.n81.2016.1562 


\section{Diego Alvarado Melitón}

e-mail: ocupacional fray@outlook.com

Nacido en el estado de México, el 12 de marzo del año

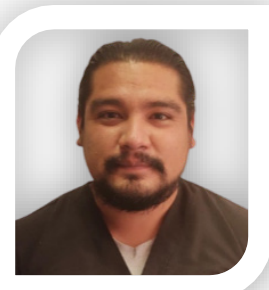

1989. Egresado de la Universidad Autónoma del estado de México (UAEM), con el título de licenciado en terapia ocupacional; y con una Maestría en docencia para nivel superior en la Universidad Tres Culturas (UTC); actualmente me desempeño como licenciado en terapia ocupacional en el manejo de pacientes psiquiátricos del Hospital Psiquiátrico Fray Bernardino Álvarez (HPFBA).

El contenido de este manuscrito se difunde bajo una Licencia de Creative Commons ReconocimientoNoComercial-Compartirlgual 4.0 Internacional 